\title{
Three Logistic Predictive Models for the Prediction of Mortality and Major Pulmonary Complications after Cardiac Surgery
}

\author{
Elena Bignami ( $\square$ elenagiovanna.bignami@unipr.it) \\ Anesthesiology, Critical Care and Pain Medicine Division, Department of Medicine and Surgery, University of Parma, Viale Gramsci 14, 43126 Parma, \\ Italy \\ Marcello Guarnieri \\ Department of Medicine and Surgery, University of Milan-Bicocca, Monza, Italy \\ Ilaria Giambuzzi \\ Department of Cardiovascular Surgery, Centro Cardiologico Monzino-IRCCS, Milan, Italy \\ Cinzia Trumello \\ Department of Cardiac Surgery, IRCCS San Raffaele Scientific Institute, Milan, Italy. \\ Francesco Saglietti \\ Department of Medicine and Surgery, University of Milan-Bicocca, Monza, Italy \\ Stefano Gianni \\ Department of Medicine and Surgery, University of Milan-Bicocca, Monza, Italy \\ Igor Belluschi \\ Department of Cardiac Surgery, IRCCS San Raffaele Scientific Institute, Milan, Italy. \\ Nora Di Tomasso \\ Department of Anesthesia and Intensive Care, IRCCS San Raffaele Scientific Institute, Milan, Italy \\ Daniele Corti \\ Department of Anesthesia and Intensive Care, IRCCS San Raffaele Scientific Institute, Milan, Italy \\ Ottavio Alfieri \\ Department of Cardiac Surgery, IRCCS San Raffaele Scientific Institute, Milan, Italy. \\ Marco Gemma \\ Department of anesthesia and intensive care, Ospedale Fatebenefratelli, Via G.B Grassi, 74 . 20157 - Milan, Italy
}

\section{Research Article}

Keywords: Cardiac anesthesia, mortality, postoperative pulmonary complications, $\mathrm{PaO2} / \mathrm{FiO} 2$.

Posted Date: January 18th, 2021

DOI: https://doi.org/10.21203/rs.3.rs-144169/v1

License: (c) (i) This work is licensed under a Creative Commons Attribution 4.0 International License. Read Full License 


\section{Abstract}

\section{Background}

Pulmonary complications after cardiac surgery are a leading cause of morbidity and mortality. The aim of this study was to develop predictive models for postoperative lung dysfunction and mortality.

\section{Methods}

This was a single-center, observational, retrospective study, which took place in a tertiary care University Hospital. We retrospectively analyzed data regarding 11285 adult patients who underwent all types of cardiac surgery from 2003 to 2015.

We developed logistic predictive models for in-hospital mortality, for postoperative pulmonary complications (PPC) occurring in the intensive care unit (ICU), and the need for postoperative non-invasive mechanical ventilation (NIMV) when clinically indicated.

\section{Results}

Age, preoperative ejection fraction, New York Heart Association (NYHA) class, emergency surgery, pharmacological and mechanical circulatory support, creatinine peak in the ICU, tracheostomy, and a lower $\mathrm{PaO}_{2} / \mathrm{FiO}_{2}$ ratio at ICU discharge were predictors of mortality. Age, ejection fraction, body mass index, creatinine peak in the ICU, blood-product use, inotropic support, and a lower $\mathrm{PaO}_{2} / \mathrm{FiO}_{2}$ ratio were predictors of postoperative NIMV. Preoperative lung disease, ejection fraction, NYHA class, inotropic and mechanical support, and preoperative serum creatinine were predictors of PPC.

\section{Conclusions}

We provide three models for the prediction of major pulmonary complications and mortality following cardiac surgery.

\section{Background}

Postoperative pulmonary complications (complete definition in Supplementary 1) are still a leading cause of morbidity after cardiac surgery, causing longer hospital and ICU length-of-stay[1], higher mortality, and increased costs[2-7].

Various factors, e.g. an inflammatory response following cardiopulmonary bypass, transfusions[8-10], ventilation stop, perfusion stop, myocardial damage, and hyperoxia[11] can all contribute to lung injury after cardiac surgery[12]. Moreover, many risk factors for lung dysfunction and prolonged mechanical ventilation after cardiac surgery have been identified; these include preoperative variables (e.g. age, sex, cardiovascular risk factors, chronic lung disease, chronic kidney disease, and coexisting endocarditis), intraoperative variables (e.g. type of surgery, pump time, intervention time, transfusions, and bleeding), and postoperative variables (e.g. inotrope dependency, and cardiogenic shock)[13-20]. Pulmonary damage has also been associated with the necessity for non-invasive mechanical ventilation (NIMV) and re-intubation, as well as with readmission to the ICU[21, 22].

The $\mathrm{PaO}_{2} / \mathrm{FiO}_{2}$ ratio is a widely used and helpful tool, especially in non-cardiac surgery, for the assessment of lung injury[23, 24]. However, few studies have investigated the predictive power of this parameter in cardiac surgery[4]. Moreover, predictive models with strong clinical implications for perioperative care are still lacking[7], especially for cardiac surgery and ICU.

The aim of this study was to investigate the predictive factors for three distinct end-points, namely in-hospital mortality, the necessity of NIMV, and the incidence of PPC after cardiac surgery. Since different potentially predictive variables become observable in different moments during the patients' clinical course, for each end-point we built a first model that considered only variables available upon hospital admission ("admission model") and a second model that considers also variables that become available at the time of surgery ("surgery model"). For in-hospital mortality and the necessity of NIMV, we could also build a third model, which also considered variables that become available at the time of ICU stay ("ICU model"). This approach is meant to provide prognostic tools applicable by clinicians during different stages of patients' clinical course.

We also studied a new cut-off in the $\mathrm{PaO}_{2} / \mathrm{FiO}_{2}$ ratio to predict the need for NIMV in the ward[25].

The main hypothesis of this study was that the $\mathrm{P}_{\mathrm{a}} \mathrm{O}_{2} / \mathrm{F}_{\mathrm{i}} \mathrm{O}_{2}$ ratio at the discharge from ICU would be a good predictor of postoperative pulmonary complications and death after cardiac surgery.

\section{Methods}

This was a single-center, observational, retrospective study that took place at a university hospital in Italy from 2003 to 2015 . The study protocol conforms to the ethical guidelines of the 1975 Declaration of Helsinki[26]. We carried out a retrospective analysis of clinical and administrative data. Following approval of the Ethics Committee of the San Raffaele IRCCS Scientific Institute (date of approval 29th October 2015), 11285 consecutive adult patients, American Society of Anesthesiologists (ASA) physical status class II or higher, who underwent open-heart surgery with or without CPB and with or without aortic cross-clamping, were included in this study. Each patient gave written informed consent. Every type of surgical intervention was included in this study. The routine perioperative management of our center is summarized in Supplementary 2[14, 27, 28]. 


\section{Statistical analysis}

Statistical analysis was carried out using the dedicated software Stata 11.1 (Copyright 2009 Stata Corp. LP, Stata Corp, 4905 Lakeway Drive, College Station, Texas 77845 USA). Categorical variables are reported as numbers (percentage). Continuous variables are reported as mean \pm SD. Their normal distribution was confirmed by both the Shapiro-Wilk W test for normality and the Kolmogorov-Smirnov tests of the equality of distributions.

We developed three logistic predictive models for in-hospital mortality (as a dichotomous variable): a first model ("preoperative model") in which variable selection was applied only to variables available at the time of hospital admission, a second model ("surgery model") considering also variables that become available at the time of surgery, and a third model ("ICU model") considering also variables that become available at the time of ICU admission. In a similar way, we developed three logistic predictive models indicating the need for postoperative NIMV (as a dichotomous variable). Moreover, for the occurrence of any PPC in the ICU (as a dichotomous variable), we developed two logistic predictive models (A "preoperative model" and a "surgery model"). Variable selection was performed with a stepwise method with probability to enter 0.10 and probability to exit 0.20 .

Model accuracy was compared by generating non-parametric ROC curves for each model and comparing their AUROC with the algorithm proposed by DeLong ER et al.28 AUROCs are reported as AUROC (95\% Cl). Model calibration was assessed reporting the intercept ("calibration-in-the-large") and the slope of the relevant calibration plots. ${ }^{26}$ Marginal predictions were used for the calculation of overall validation measures. ${ }^{29}$

Since the $\mathrm{PaO} 2 / \mathrm{FiO} 2$ ratio entered our final predictive models both for in-hospital mortality and NIMV use and given its widespread use as a predictor thereof, we studied the relevant ROC curves to report the PaO2/FiO2 cut-off value that maximizes sensitivity and specificity.

Our series covers a wide time span, so the year of surgery was considered as a random effect variable and the aforementioned analysis models were actually studied as mixed logistic regression models. The difference between mixed versus logistic regression models was tested with the Loglikelihood ratio test. The Intra cluster Correlation Coefficient (ICC) is reported for each mixed effect model. The random intercepts were omitted from the prediction equation to provide risk estimates for "an average" year, which is clinically useful when addressing prediction for years, not in our sample.

Moreover, given the high number of cases in our series, we split the series into a training set and a test set. The aforementioned models were obtained on the training set and were tested thereafter on the test set. The training set was obtained by selecting cases from our original series with a Bernoulli process (random selection without replacement) with probability 0.66: this generated a training set containing 7448 cases. The test set consisted of the 3837 cases not selected in the training set. The AUROC of the models applied to the test sets is reported together with their $95 \% \mathrm{Cl}$ and compared with the corresponding training test AUROCs with the Chi-square test.

We developed three logistic predictive models for in-hospital mortality (as a dichotomous variable). In the first model ("Admission model"), the predictive variable selection was performed on variables with data available on admission, i.e. age (years), surgical diagnosis, coronaropathy (yes/no), ACEF score value[28] (\%), BMI ( $\left.\mathrm{kg} / \mathrm{m}^{2}\right)$, redo surgery (yes/no), elective surgery (yes/no), hypertension (yes/no), diabetes mellitus (yes/no), chronic obstructive pulmonary disease (yes/no), peripheral vasculopathy (yes/no), previous ictus or transitory ischemic attack (yes/no), preoperative history of smoking (yes/no), preoperative ejection fraction (\%), preoperative serum creatinine (mg/dl), New York Heart Association class (from I to IV), ASA class (from I to V). In the second model ("Surgery model"), variables with data available after completion of surgery were added to the variable selection process, i.e. aortic cross-clamp (yes/no) and aortic cross-clamp time (minutes), blood-product administration during surgery (yes/no), inotropic support during surgery (yes/no), and use of Intra-Aortic Balloon Pump (IABP) (yes/no). In the third model ("ICU model"), variables with data available from ICU stay were added to the variable selection process, i.e. peak postoperative creatinine in ICU (mg/dl), the lowest hematocrit value in ICU (\%), inotropic drugs after surgery (yes/no), postoperative IABP (yes/no), duration of mechanical ventilation (hours), postoperative renal replacement therapy (yes/no), postoperative Extracorporeal Membrane Oxygenator (ECMO) (yes/no), tracheostomy in ICU (yes/no), re-intubation in ICU (yes/no), postoperative pulmonary complication (PPC[14]) (yes/no), septic shock (yes/no, according to the Sepsis-3 consensus definition[27]), use of diuretic therapy (yes/no), blood-product administration during ICU stay (yes/no), $\mathrm{PaO}_{2} / \mathrm{FiO}_{2}$ ratio on ICU discharge.

Similarly, we developed three logistic predictive models indicating the need for postoperative NIMV (as a dichotomous variable). Moreover, we developed two logistic predictive models for the occurrence of any PPC during ICU stay (as a dichotomous variable).

In the variable selection process, variables with $\mathrm{P}<0.20$ were taken as possible predictors for the multivariate model. When developing the final model, $\mathrm{P}$ $<0.05$ was considered significant. The interaction was checked, and linearity in the logit was verified using the fractional polynomial method.

In reporting the final models, odds ratios are shown as OR $(95 \% \mathrm{Cl})$.

We included under the definition of NIMV all the techniques which involved the application of a positive pressure via a face mask or helmet, mostly including a continuous positive airway pressure (CPAP) and a pressure support ventilation with positive end-expiratory pressure (PEEP). The main indication for CPAP was hypoxemic respiratory failure of any origin, defined as $\mathrm{PaO}_{2} / \mathrm{FiO}_{2}$ ratio between 100 and 250 . The main indication for pressure support NIMV was respiratory acidosis.

\section{Sample size calculation}


Given the retrospective nature of our study, no formal prior sample size evaluation was performed. Nevertheless in building our logistic regression models we took care to respect the well-known rule of thumb derived from simulation studies of a minimum of 10 events per dependent variable.

\section{Results}

The study population includes 11285 and is described in Table 1.

Table 1

Description of the baseline data of the study population $(\mathrm{N}=11285)$. Data are expressed as mean \pm standard deviation or number (percentage). Preoperative chronic obstructive pulmonary disease (COPD) was defined according to clinical criteria (a patient was considered to be affected by COPD

if he was prescribed inhalation bronchodilators or corticosteroids or other drugs labeled for COPD therapy by a pneumologist of the family physician, even without obtaining confirmation from

\begin{tabular}{|c|c|}
\hline Variable & Preoperative value \\
\hline Age, y & $67.55 \pm 13.97$ \\
\hline Male sex, n (\%) & $9844(87.2 \%)$ \\
\hline Height, cm & $169 \pm 9$ \\
\hline Weight, kg & $73 \pm 13$ \\
\hline $\mathrm{BMI}, \mathrm{kg} / \mathrm{m}^{2}$ & $25.46 \pm 3.95$ \\
\hline Preoperative EF, \% & $56.41 \% \pm 9.77$ \\
\hline \multicolumn{2}{|l|}{ NYHA class > II, n (\%) } \\
\hline \multicolumn{2}{|l|}{ Preoperative comorbidities } \\
\hline COPD, n (\%) & $920(8.1 \%)$ \\
\hline Hypertension, n (\%) & $7.248(64.2 \%)$ \\
\hline Type II Diabetes, n (\%) & $1.528(13.5 \%)$ \\
\hline Preoperative creatinine, mg/dl & $0.98 \pm 0.67$ \\
\hline Chronic renal failure, n (\%) & $1161(10.2 \%)$ \\
\hline Peripheral vasculopathy, n (\%) & $2036(18.0 \%)$ \\
\hline Smoking habits, n (\%) & $2443(21.6 \%)$ \\
\hline Stroke, n (\%) & $989(8.8 \%)$ \\
\hline \multicolumn{2}{|l|}{ Timing of surgery } \\
\hline Emergency or urgency, $\mathrm{n}(\%)$ & $214(1.9 \%)$ \\
\hline Planned, n (\%) & $11071(98.1 \%)$ \\
\hline \multicolumn{2}{|l|}{ Surgery type } \\
\hline Valvular surgery, n (\%) & $5178(45.88 \%)$ \\
\hline Coronary surgery, n (\%) & $1814(16.07 \%)$ \\
\hline Ascending aorta aneurysm surgery, $\mathrm{n}(\%)$ & $420(3.71 \%)$ \\
\hline Other surgical procedures, n (\%) & $999(8.85 \%)$ \\
\hline Combined surgery (two or more procedures), $\mathrm{n}(\%)$ & $5564(49.31 \%)$ \\
\hline
\end{tabular}

The most frequent $(5564,49.31 \%)$ procedure was combined surgery (Coronary Artery Bypass Graft + valvular procedure), followed by valvular surgery alone $(5178,45.88 \%)$. Coronary surgery alone was performed on 1814 patients (16.07\%). Aortic surgery was performed on 420 patients (3.71\%). The remaining 999 patients (8.85\%) underwent other cardiac surgery procedures, such as percutaneous surgery (e.g. Mitraclip ${ }^{\circledR}$ implantation), tumor exeresis, the Maze procedure for atrial fibrillation, or patent foramen ovale closure. The PaO2/FiO2 ratio at ICU discharge was $292.6 \pm 114.0$, showing normal distribution. The initial population's mean age was 67.55 years \pm 13.97 . Mean BMl was $25.46 \mathrm{~kg} / \mathrm{m}^{2} \pm 3.95$. The mean preoperative ejection fraction was $56.41 \% \pm 9.77$ 
Intraoperative inotropic support was necessary for 5339 patients (47.31\%). Postoperative pulmonary complications occurred in 248 (2.19\%) cases. Non-Invasive Mechanical Ventilation, considering both continuous positive airway pressure (CPAP) and pressure support ventilation (PSV), was prescribed in 658 patients $(5.83 \%)$.

\section{In-hospital mortality predictive models}

\subsection{Preoperative model}

Four variables predicted in-hospital mortality in the preoperative multivariate logistic model (whole model $P<0.0001 ; P s e u d o ~ R{ }^{2}=0.10$ ), namely age, $E F$, NYHA class, and elective vs. emergency procedure. When the year of the procedure was considered as a random effect, the resulting mixed effect model did not differ from the correspondent logistic regression model (Likelihood ratio test $\mathrm{P}=1.000 ; \mathrm{ICC}<0.01$ ). Nevertheless, table 2 reports on the model which entails the year of surgery as a random intercept, given its importance.

This model's AUROC was $0.81(95 \% \mathrm{Cl}=0.76-0.85)$.

When the model was applied to the test set, its AUROC was $0.79(95 \% \mathrm{Cl}=0.75-0.83)$ and did not differ from the training test's $(P=0.6685)$. The relevant calibration plot exhibited calibration-in-the-large $=0.0024$ and calibration slope $=0.7065$.

\subsection{Surgery model}

Five variables predicted in-hospital mortality in the surgery multivariate logistic model (whole model $p<0,0001 ; P$ seudo $R^{2}=0,18$ ), namely use of inotropic drugs, use of Intra-Aortic Balloon Pump (IABP), age, NYHA class, and elective vs. emergency procedure. When the year of the procedure was considered as a random effect, the resulting mixed effect model did not differ from the correspondent logistic regression model (Likelihood ratio test $\mathrm{P}=0,32 ; \mathrm{ICC}=0,02)$. Nevertheless, Table2 reports on the model, which entails the year of surgery as a random intercept, given its importance.

This model's AUROC was $0,86(95 \% \mathrm{Cl}=0,82-0,89)$. When the model was applied to the test set, its AUROC was $0,85(95 \% \mathrm{Cl}=0,82-0,89)$ : this did not differ from the training test's $(P=0,7417)$, but was significantly higher than the corresponding test admission model's AUROC $(P=0,0005)$. The relevant calibration plot exhibited calibration-in-the-large=0,0057 and calibration slope=0,8643 (see figure 1).

\subsection{ICU model}

Six variables predicted in-hospital mortality in the ICU multivariate logistic model (whole model $p<0,0001 ; P$ seudo $R^{2}=0,26$ ), namely serum creatinine peak value in ICU, tracheostomy, use of inotropic drugs, NYHA class, age, and $\mathrm{PaO}_{2} / \mathrm{FiO}_{2}$ at ICU discharge. When the year of the procedure was considered as a random effect, the resulting mixed effect model did not differ from the correspondent logistic regression model (Likelihood ratio test $P=1,000 ; I C C=0,11)$. Nevertheless, Table 2 reports on the model which entails the year of surgery as a random intercept, given its importance.

This model's AUROC was $0,90(95 \% \mathrm{Cl}=0,87-0,94)$. When the model was applied to the test set, its $\mathrm{AUROC}$ was $0,89(95 \% \mathrm{Cl}=0,84-0,93)$ and did not differ from the training test's $(P=0,4390)$, but was significantly higher than the corresponding test surgery model's AUROC ( $P=0,0061)$. The relevant calibration plot exhibited calibration-in-the-large=0,0021 and calibration slope=0,8443.(see Figure 1 )

\section{Postoperative NIMV predictive models}

\subsection{Preoperative /Surgery model}

No variable with data available after surgery entered a multivariate model. Hence the "Preoperative" and "Surgery" multivariate logistic models for NIMV use were actually not different from one another.

Four variables predicted NIMV use in the preoperative/surgery multivariate logistic model (whole model $P<0,0001 ; P$ seudo $\left.R^{2}=0,05\right)$, namely age, $E F$, Body Mass Index (BMI), and preoperative serum creatinine. When the year of the procedure was considered as a random effect, the resulting mixed effect model differed from the correspondent logistic regression model (Likelihood ratio testP=0.0002; ICC $=0.08$ ). Tab. 2 reports on the model which entails the year of surgery as a random intercept.

This model's AUROC was $0.71(95 \% \mathrm{Cl}=0.67-0.75)$.

When the model was applied to the test set, its AUROC was $0.71(95 \% \mathrm{Cl}=0.67-0.75)$ and did not differ from the training test's $(\mathrm{P}=0.8687)$. The relevant calibration plot exhibited calibration-in-the-large $=0.0057$ and calibration slope $=0.8643$. 
Table 2

Results of the logistic regression models.

\begin{tabular}{|c|c|c|c|c|c|c|c|c|c|c|c|c|}
\hline \multirow[b]{2}{*}{$\begin{array}{l}\text { Preoperative } \\
\text { models }\end{array}$} & \multicolumn{4}{|c|}{ Models for mortality } & \multicolumn{4}{|c|}{ Models for NIMV } & \multicolumn{4}{|c|}{ Models for PPC } \\
\hline & $\begin{array}{l}\text { Predictive } \\
\text { variable }\end{array}$ & $\begin{array}{l}\text { Odds } \\
\text { ratio }\end{array}$ & $\begin{array}{l}95 \% \\
\mathrm{Cl}\end{array}$ & $\begin{array}{l}\mathrm{P}- \\
\text { value }\end{array}$ & $\begin{array}{l}\text { Predictive } \\
\text { variable }\end{array}$ & $\begin{array}{l}\text { Odds } \\
\text { ratio }\end{array}$ & $\begin{array}{l}95 \% \\
\mathrm{Cl}\end{array}$ & $\begin{array}{l}\mathrm{P} \text { - } \\
\text { value }\end{array}$ & $\begin{array}{l}\text { Predictive } \\
\text { variable }\end{array}$ & $\begin{array}{l}\text { Odds } \\
\text { ratio }\end{array}$ & $\begin{array}{l}95 \% \\
\mathrm{Cl}\end{array}$ & $\begin{array}{l}\mathrm{P}- \\
\text { value }\end{array}$ \\
\hline & Age & 1.05 & $\begin{array}{l}1.02- \\
1.08\end{array}$ & $<0.001$ & Age & 1.04 & $\begin{array}{l}1.02- \\
1.06\end{array}$ & $<0.001$ & COPD & 2.63 & $\begin{array}{l}1.31 \\
-5.28\end{array}$ & 0.007 \\
\hline & $\begin{array}{l}\text { Preoperative } \\
\text { EF }\end{array}$ & 0.97 & $\begin{array}{l}0.95- \\
0.99\end{array}$ & 0.011 & $\begin{array}{l}\text { Preoperative } \\
\text { EF }\end{array}$ & 0.97 & $\begin{array}{l}0.96- \\
1.00\end{array}$ & 0.023 & $\begin{array}{l}\text { Creatinine } \\
\text { Peaks }\end{array}$ & 1.48 & $\begin{array}{l}1.19 \\
-1.83\end{array}$ & $<0.001$ \\
\hline & NYHA class & 2.97 & $\begin{array}{l}1.63- \\
5.41\end{array}$ & $<0.001$ & BMI & 1.10 & $\begin{array}{l}1.05- \\
1.15\end{array}$ & $<0.001$ & $\mathrm{EF}$ & 0.97 & $\begin{array}{l}0.95 \\
- \\
0.99\end{array}$ & 0.004 \\
\hline & $\begin{array}{l}\text { Elective } \\
\text { surgery }\end{array}$ & 0.29 & $\begin{array}{l}0.90- \\
0.91\end{array}$ & 0.036 & $\begin{array}{l}\text { Preoperative } \\
\text { Creatinine }\end{array}$ & 1.26 & $\begin{array}{l}1.01- \\
1.58\end{array}$ & 0.043 & NYHA class & 1.81 & $\begin{array}{l}1.05 \\
- \\
3.14\end{array}$ & 0.033 \\
\hline & $\begin{array}{l}\text { Random } \\
\text { effect variable }\end{array}$ & $S D$ & $S E$ & $P$ & $\begin{array}{l}\text { Random } \\
\text { effect } \\
\text { variable }\end{array}$ & $S D$ & $S E$ & $P$ & $\begin{array}{l}\text { Random } \\
\text { effect } \\
\text { variable }\end{array}$ & $S D$ & $S E$ & $P$ \\
\hline & $\begin{array}{l}\text { Year of } \\
\text { surgery }\end{array}$ & $<0.001$ & 0.37 & 1.000 & $\begin{array}{l}\text { Year of } \\
\text { surgery }\end{array}$ & 0.53 & 0.18 & $<0.001$ & $\begin{array}{l}\text { Year of } \\
\text { surgery }\end{array}$ & 0.28 & 0.20 & 0.176 \\
\hline \multirow[t]{8}{*}{$\begin{array}{l}\text { Surgery } \\
\text { models }\end{array}$} & $\begin{array}{l}\text { Predictive } \\
\text { variable }\end{array}$ & $\begin{array}{l}\text { Odds } \\
\text { ratio }\end{array}$ & $\begin{array}{l}95 \% \\
\mathrm{Cl}\end{array}$ & $\begin{array}{l}\mathrm{P}- \\
\text { value }\end{array}$ & $\begin{array}{l}\text { Predictive } \\
\text { variable }\end{array}$ & $\begin{array}{l}\text { Odds } \\
\text { ratio }\end{array}$ & $\begin{array}{l}95 \% \\
\mathrm{Cl}\end{array}$ & $\begin{array}{l}\mathrm{P}- \\
\text { value }\end{array}$ & $\begin{array}{l}\text { Predictive } \\
\text { variable }\end{array}$ & $\begin{array}{l}\text { Odds } \\
\text { ratio }\end{array}$ & $\begin{array}{l}95 \% \\
\mathrm{Cl}\end{array}$ & $\begin{array}{l}\mathrm{P}- \\
\text { value }\end{array}$ \\
\hline & $\begin{array}{l}\text { Inotropes in } \\
\text { the operating } \\
\text { room }\end{array}$ & 3.09 & $\begin{array}{l}1.45 \\
-6.6\end{array}$ & 0.003 & Age & 1.04 & $\begin{array}{l}1.02- \\
1.06\end{array}$ & $<0.001$ & $\begin{array}{l}\text { Inotropes in } \\
\text { the } \\
\text { operating } \\
\text { room }\end{array}$ & 2.79 & $\begin{array}{l}1.38- \\
5.64\end{array}$ & 0.004 \\
\hline & $\begin{array}{l}\text { IABP in the } \\
\text { operating } \\
\text { room }\end{array}$ & 3.91 & $\begin{array}{l}1.90 \\
- \\
8.04\end{array}$ & $<0.001$ & $\begin{array}{l}\text { Preoperative } \\
\text { EF }\end{array}$ & 0.97 & $\begin{array}{l}0.96- \\
1.00\end{array}$ & 0.023 & $\begin{array}{l}\text { IABP in the } \\
\text { operating } \\
\text { room }\end{array}$ & 2.64 & $\begin{array}{l}1.02- \\
6.81\end{array}$ & 0.045 \\
\hline & Age & 1.06 & $\begin{array}{l}1.03 \\
- \\
1.10\end{array}$ & $<0.001$ & BMI & 1.10 & $\begin{array}{l}1.05- \\
1.15\end{array}$ & $<0.001$ & COPD & 3.74 & $\begin{array}{l}1.64- \\
8.51\end{array}$ & 0.002 \\
\hline & NYHA class & 2.35 & $\begin{array}{l}1.24 \\
- \\
4.47\end{array}$ & 0.009 & $\begin{array}{l}\text { Preoperative } \\
\text { Creatinine }\end{array}$ & 1.26 & $\begin{array}{l}1.01- \\
1.58\end{array}$ & 0.043 & $\begin{array}{l}\text { Preoperative } \\
\text { creatinine }\end{array}$ & 1.39 & $\begin{array}{l}1.07- \\
1.81\end{array}$ & 0.014 \\
\hline & $\begin{array}{l}\text { Elective } \\
\text { surgery }\end{array}$ & 0.22 & $\begin{array}{l}0.08 \\
- \\
0.65\end{array}$ & 0.006 & & & & & & & & \\
\hline & $\begin{array}{l}\text { Random } \\
\text { effect variable }\end{array}$ & $S D$ & $S E$ & $P$ & $\begin{array}{l}\text { Random } \\
\text { effect } \\
\text { variable }\end{array}$ & $S D$ & $S E$ & $P$ & $\begin{array}{l}\text { Random } \\
\text { effect } \\
\text { variable }\end{array}$ & $S D$ & $S E$ & $P$ \\
\hline & $\begin{array}{l}\text { Year of } \\
\text { surgery }\end{array}$ & 0.24 & 0.30 & 0.320 & $\begin{array}{l}\text { Year of } \\
\text { surgery }\end{array}$ & 0.53 & 0.18 & $<0.001$ & $\begin{array}{l}\text { Year of } \\
\text { surgery }\end{array}$ & 0.53 & 0.25 & 1.329 \\
\hline \multirow[t]{7}{*}{ ICU models } & $\begin{array}{l}\text { Predictive } \\
\text { variable }\end{array}$ & $\begin{array}{l}\text { Odds } \\
\text { ratio }\end{array}$ & $\begin{array}{l}95 \% \\
\mathrm{Cl}\end{array}$ & $\begin{array}{l}\mathrm{P}- \\
\text { value }\end{array}$ & $\begin{array}{l}\text { Predictive } \\
\text { variable }\end{array}$ & $\begin{array}{l}\text { Odds } \\
\text { ratio }\end{array}$ & $\begin{array}{l}95 \% \\
\mathrm{Cl}\end{array}$ & $\begin{array}{l}\mathrm{P}- \\
\text { value }\end{array}$ & & & & \\
\hline & $\begin{array}{l}\text { Creatinine } \\
\text { peak }\end{array}$ & 1.50 & $\begin{array}{l}1.24 \\
- \\
1.82\end{array}$ & $<0.001$ & $\begin{array}{l}\text { Creatinine } \\
\text { peak }\end{array}$ & 1.35 & $\begin{array}{l}1.21 \\
-1.51\end{array}$ & $<0.001$ & & & & \\
\hline & Tracheostomy & 18.08 & $\begin{array}{l}7.14 \\
- \\
45.76\end{array}$ & $<0.001$ & Inotropes & 1.60 & $\begin{array}{l}1.25 \\
- \\
2.04\end{array}$ & $<0.001$ & & & & \\
\hline & $\begin{array}{l}\text { Inotropes in } \\
\text { the ICU in the } \\
\text { ICU }\end{array}$ & 2.52 & $\begin{array}{l}1.01- \\
5.77\end{array}$ & 0.029 & $\mathrm{P} / \mathrm{F}$ & 0.99 & $\begin{array}{l}0.991- \\
0.993\end{array}$ & $<0.001$ & & & & \\
\hline & NYHA class & 2.79 & $\begin{array}{l}1.35 \\
- \\
5.78\end{array}$ & 0.006 & $\begin{array}{l}\text { Blood } \\
\text { transfusion }\end{array}$ & 2.41 & $\begin{array}{l}1.87 \\
- \\
3.13\end{array}$ & $<0.001$ & & & & \\
\hline & Age & 1.08 & $\begin{array}{l}1.03 \\
-1.12\end{array}$ & $<0.001$ & BMI & 1.07 & $\begin{array}{l}1.05 \\
-1.11\end{array}$ & $<0.001$ & & & & \\
\hline & $\mathrm{P} / \mathrm{F}$ ratio & 0.1 & $\begin{array}{r}0.99 \\
-0.1\end{array}$ & 0.028 & & & & & & & & \\
\hline
\end{tabular}




\begin{tabular}{|c|c|c|c|c|c|c|c|}
\hline $\begin{array}{l}\text { Random } \\
\text { effect variable }\end{array}$ & $S D$ & $S E$ & $P$ & $\begin{array}{l}\text { Random } \\
\text { effect } \\
\text { variable }\end{array}$ & $S D$ & $S E$ & $P$ \\
\hline $\begin{array}{l}\text { Year of } \\
\text { surgery }\end{array}$ & $<0.001$ & 0.44 & 1.000 & $\begin{array}{l}\text { Year of } \\
\text { surgery }\end{array}$ & 0.83 & 0.18 & $<0.001$ \\
\hline \multicolumn{8}{|c|}{ List of abbreviations used } \\
\hline
\end{tabular}

\subsection{ICU model}

Four variables predicted NIMV use in the ICU multivariate logistic model (whole model $p<0.0001$; Pseudo $\mathrm{R}^{2}=0.13$ ), namely serum creatinine peak in ICU, inotropic drug use, $\mathrm{P}_{\mathrm{a}} \mathrm{O}_{2} / \mathrm{F}_{\mathrm{i}} \mathrm{O}_{2}$ ratio at ICU discharge, use of blood products, and $\mathrm{BMI}$. When the year of the procedure was considered as a random effect, the resulting mixed effect model (both intercept and slope) differed from the correspondent logistic regression model (Likelihood ratio test $\mathrm{P}<0.001 ; \mathrm{ICC}=0.17)$. Tab. 2 reports on the model which entails the year of surgery as a random intercept.

This model's AUROC was $0.81(95 \% \mathrm{Cl}=0.77-0.85)$. When the model was applied to the test set, its AUROC was $0.79(95 \% \mathrm{Cl}=0.77-0.81)$ and did not differ from the training test's $(P=0.3966)$ but was significantly higher than the corresponding test surgery model's AUROC $(P<0.0001)$. The relevant calibration plot exhibited calibration-in-the-large $=0.0063$ and calibration slope $=0.8814$.(see Figure 2 )

\section{Postoperative pulmonary complication predictive model}

\subsection{Preoperative model}

Four variables predicted PPC in the preoperative multivariate logistic model (whole model $\mathrm{P}<0.00001$; Pseudo $\mathrm{R}^{2}=0.06$ ), namely Chronic Obstructive Pulmonary Disease (COPD), preoperative serum creatinine, EF, and NYHA class. When the year of the procedure was considered as a random effect, the resulting mixed effect model did not differ from the correspondent logistic regression model (Likelihood ratio test $P=0.1767 ;$ ICC $=0.06$ ). Nevertheless, Tab. 2 reports on the model which entails the year of surgery as a random intercept, given its importance

This model's AUROC was $0.70(95 \% \mathrm{Cl}=0.62-0.78)$.

When the model was applied to the test set, its AUROC was $0.69(95 \% \mathrm{Cl}=0.61-0.76)$ and did not differ from the training test's $(\mathrm{P}=0.9299)$. The relevant calibration plot exhibited calibration-in-the-large $=0.0059$ and calibration slope $=0.5278$.

\subsection{Surgery model}

Four variables predicted PPC in the Surgery multivariate logistic model (whole model $p<0.0001$; Pseudo $R^{2}=0.088$ ), namely use of inotropic drugs, use of IABP, COPD, and preoperative serum creatinine. When the year of the procedure was considered as a random effect, the resulting mixed effect model (both intercept and slope) differed from the correspondent logistic regression model (Likelihood ratio test $\mathrm{P}=0.0275$; ICC $=0.08$ ). Tab. 2 reports on the model which entails the year of surgery as a random intercept.

This model's AUROC was $0.70(95 \% \mathrm{Cl}=0.62-0.78)$. When the model was applied to the test set, its AUROC was $0.68(95 \% \mathrm{Cl}=0.60-0.76)$ and did not differ from the training test's $(P=0.3478)$, but was not significantly different from the corresponding test admission model's AUROC ( $P=0.4467)$. The relevant calibration plot exhibited calibration-in-the-large $=-0.0006$ and calibration slope=1.1434.(see Figure 1)

\section{$4 \mathrm{~A}$ new cut-off for the $\mathrm{PaO}_{2} / \mathrm{FiO}_{2}$ ratio}

A ROC curve was developed using the $\mathrm{PaO}_{2} / \mathrm{FiO}_{2}$ ratio at ICU discharge and the incidence of NIMV use during hospital stay. The PaO2/FiO2 ratio cut-off value, maximizing sensitivity, and specificity was 240 . At this point in the curve, sensitivity was $66,53 \%$ while specificity was $66,06 \%$, correct classification occurred in $66.51 \%$ of cases. Figure 2 shows the ROC curve we developed.

\section{Discussion}

We investigated the potential predictive factors for mortality and NIMV use after cardiac surgery in a large adult population (11285 patients undergoing elective or emergency cardiac surgery). Our model found significant predictors among many pre-operative, intraoperative, and post-operative factors (Table 2). 
We propose a new cut-off for $\mathrm{PaO}_{2} / \mathrm{FiO}_{2}$ ratio evaluation. The ROC curve we developed found the best cut-off point that maximized sensitivity and specificity at 239. The curve was developed on a cohort of 11285 patients. In what might be referred to as the gray zone, this information might help clinicians to distinguish between those patients with sub-optimal gas exchange that do not require respiratory support and those that do need it.

The discharge from the ICU must be safe[29]. Cardiac-surgery patients with risk factors for postoperative respiratory insufficiency would probably benefit from closer monitoring of gas exchange and respiratory mechanics.

Among anamnestic data, we underlined the importance of age, poor cardiac function with symptoms of heart failure (quantified using the NYHA classification), poor renal function, the presence of COPD, and obesity. Intraoperative low cardiac output, highlighted by the need for inotropic support and IABP, is shown by our data to be a predictor of poor outcome regarding pulmonary complications. During post-operative care, attention should be paid to patients receiving high quantities of blood products, which can lead to a risk of direct lung injury[21, 29].

Our data show the association between inotropic and mechanical circulatory support and lung dysfunction, as well as mortality. We believe that hemodynamic instability and low cardiac output syndrome are implicated in these findings, although inotropic drugs have their own side-effects as they increase myocardial oxygen consumption.

All of these risk factors need to be taken into consideration during clinical decision-making, and patients at high risk of postoperative pulmonary dysfunction can benefit from a specific protocol for intraoperative and postoperative optimization, for ICU discharge, and for respiratory chest physiotherapy and NIMV in the ward. In addition to the previously described risk factors, this might help to decide optimal ICU discharge timing. The delayed discharge could lead to healthcare-related infections and is globally associated with a worse outcome[19] and increased costs, whereas premature discharge carries the risk of readmission to the ICU, which can also be very harmful[22].

Other studies have investigated post-cardiac-surgery pulmonary complications and the predictive value of the $\mathrm{PaO}_{2} / \mathrm{FiO}{ }_{2}$ ratio. $\mathrm{A}$ prospective observational trial on 2725 consecutive cardiac-surgery patients[21] identified lower $\mathrm{PaO}_{2} / \mathrm{FiO}_{2}$ ratio values in non-survivors compared to survivors and highlighted the importance of this parameter in predicting a worse postoperative outcome. Another smaller observational trial identified several risk factors for a reduced $\mathrm{PaO}_{2} / \mathrm{FiO}_{2}$ ratio during the postoperative period, including age, obesity, reduced cardiac function, emergency surgery, high creatinine levels, and inotropic support[4].

We also identified factors associated with the need for NIMV and the incidence of postoperative pulmonary complications. We confirm data provided by a recent retrospective trial[16] regarding obesity and postoperative hypoxia, defining preoperative BMI as a predictor for postoperative NIMV, and a reduced $\mathrm{PaO}_{2} / \mathrm{FiO}_{2}$ ratio at ICU discharge. A smaller observational trial[15] identified $\mathrm{BMI}$ as an important risk factor, providing findings similar to ours concerning intraoperative risk factors for difficult respiratory management, concentrating on prolonged mechanical ventilation in the ICU. Finally, a recent study on 145 adult cardiac-surgery patients found a correlation between lower $\mathrm{PaO}_{2} / \mathrm{FiO}_{2}$ ratios and length of hospital and ICU stay[29].

The importance of our data is represented by the larger sample size compared with previous studies, the robustness of our statistical analysis, and the step-by-step inclusion of preoperative, intraoperative, and postoperative factors in a manner closely related to a clinical practice model, which might prove useful to identify high-risk patients that could benefit from closer monitoring and observation. Moreover, the need for NIMV and the presence of PPC following cardiac surgery have never been examined with a comprehensive predictive model. Finally, we were able to provide a new cut-off for $\mathrm{PaO}_{2} / \mathrm{FiO}_{2}$ ratio evaluation in routine clinical activity.

The main limitation of the present study is that it is a retrospective analysis. We were unable to collect all of the necessary data from each of the included patients as many of the source documents were 12 years old, and it was at times, difficult to obtain the correct information. Due to stepwise selection, the global sample size is numerically inferior to the initial sample size. We acknowledge that some random error may still be present in data coming from different years and multiple sources, but we are confident that the high number of patients analyzed and the robustness of the models we developed can overcome this limitation. Another important limitation is that anesthesia and surgical techniques have changed over the years, and we are aware that what we performed in 2003 may not be the same as what we are doing nowadays. Nevertheless, we believe that the risk factors we investigated, such as preoperative conditions, intraoperative inotropes and mechanical support, postoperative infections, etc. are, unfortunately, everpresent problems. The management and understanding of complications and high-risk patients might have changed, but the pathophysiology of diseases, together with the risk factors and predictors, most probably have not.

\section{Conclusions}

We define important risk factors for pulmonary complications following cardiac surgery, for NIMV use in the ICU or in the ward, and for hospital mortality. We found a new cut-off for the $\mathrm{PaO}_{2} / \mathrm{FiO}_{2}$ ratio to evaluate patients undergoing adult cardiac surgery that might prove useful for clinical decision-making.

\section{Declarations}

\section{- Ethics approval and consent to participate}

Page 8/12 
Ethics approval and consent to participate

The study was performed after the approval of the Ethics Committee of the San Raffaele IRCCS Scientific Institute (date of approval 29th October

2015). Each patient gave written informed consent.

\section{- Consent for publication}

Not applicable.

- Availability of data and materials

All data generated or analysed during this study are included in this published article [and its supplementary information files].

\section{- Competing interests}

The authors declare that they have no competing interests.

\section{- Funding}

This study was performed with departmental funds only.

\section{- Authors' contributions}

The study was designed by EB and MaGu. They also contributed to manuscript writing.

MaGe performed the data analysis, interpretation of the final results and contributed to manuscript writing.

OA contributed with interpretation of the final results and with manuscript writing.

IG, FS, SG, IB, NDT, CT and DC contributed with data collection.

All the authors approved the final manuscript submitted to this Journal.

\section{- Acknowledgements}

We want to thank Michael John, of the Vita-Salute University, for English language editing of this manuscript.

\section{References}

1. Almashrafi A, Elmontsri M, Aylin P. Systematic review of factors influencing length of stay in ICU after adult cardiac surgery. BMC Health Serv Res. 2016;16:318.

2. Magnusson L, Zemgulis V, Wicky S, Tydén H, Thelin S, Hedenstierna G. Atelectasis is a major cause of hypoxemia and shunt after cardiopulmonary bypass: an experimental study. Anesthesiology. 1997;87:1153-63.

3. Weiss YG, Merin G, Koganov E, Ribo A, Oppenheim-Eden A, Medalion B, et al. Postcardiopulmonary bypass hypoxemia: a prospective study on incidence, risk factors, and clinical significance. J Cardiothorac Vasc Anesth. 2000;14:506-13.

4. Canet J, Gallart L, Gomar C, Paluzie G, Vallès J, Castillo J, et al. Prediction of postoperative pulmonary complications in a population-based surgical cohort. Anesthesiology. 2010;113:1338-50.

5. Wynne R, Botti M. Postoperative pulmonary dysfunction in adults after cardiac surgery with cardiopulmonary bypass: clinical significance and implications for practice. Am J Crit Care. 2004;13:384-93.

6. Pizov R, Weiss YG, Oppenheim-Eden A, Glickman H, Goodman S, Koganov Y, et al. High oxygen concentration exacerbates cardiopulmonary bypass-induced lung injury. J Cardiothorac Vasc Anesth. 2000;14:519-23.

7. Bignami E, Guarnieri M, Saglietti F, Belletti A, Trumello C, Giambuzzi I, et al. Mechanical Ventilation During Cardiopulmonary Bypass. J Cardiothorac Vasc Anesth. 2016;30:1668-75.

8. Vlaar APJ, Hofstra JJ, Determann RM, Veelo DP, Paulus F, Levi M, et al. Transfusion-related acute lung injury in cardiac surgery patients is characterized by pulmonary inflammation and coagulopathy: A prospective nested case-control study*. Crit Care Med. 2012;40:2813.

9. Vlaar APJ, Cornet AD, Hofstra JJ, Porcelijn L, Beishuizen A, Kulik W, et al. The effect of blood transfusion on pulmonary permeability in cardiac surgery patients: a prospective multicenter cohort study. Transfusion . 2012;52:82-90.

10. Vlaar APJ, Hofstra JJ, Determann RM, Veelo DP, Paulus F, Kulik W, et al. The incidence, risk factors, and outcome of transfusion-related acute lung injury in a cohort of cardiac surgery patients: a prospective nested case-control study. Blood. 2011;117:4218-25. 
11. Totonchi Z, Baazm F, Chitsazan M, Seifi S, Chitsazan M. Predictors of prolonged mechanical ventilation after open heart surgery. J Cardiovasc Thorac Res. 2014;6:211-6.

12. Bartz RR, Ferreira RG, Schroder JN, Davies J, Liu W-W, Camara A, et al. Prolonged pulmonary support after cardiac surgery: incidence, risk factors and outcomes: a retrospective cohort study. J Crit Care. 2015;30:940-4.

13. Rezaianzadeh A, Maghsoudi B, Tabatabaee H, Keshavarzi S, Bagheri Z, Sajedianfard J, et al. Factors associated with extubation time in coronary artery bypass grafting patients. PeerJ. 2015;3:e1414.

14. Wynne R. Variable definitions: implications for the prediction of pulmonary complications after adult cardiac surgery. Eur $\mathrm{J}$ Cardiovasc Nurs. 2004;3:43-52.

15. Cislaghi F, Condemi AM, Corona A. Predictors of prolonged mechanical ventilation in a cohort of 3,269 CABG patients. Minerva Anestesiol. 2007;73:615-21.

16. Cislaghi F, Condemi AM, Corona A. Predictors of prolonged mechanical ventilation in a cohort of 5123 cardiac surgical patients. Eur $\mathrm{J}$ Anaesthesiol. 2009;26:396-403.

17. Ji Q, Mei Y, Wang X, Feng J, Cai J, Ding W. Risk factors for pulmonary complications following cardiac surgery with cardiopulmonary bypass. Int J Med Sci. 2013;10:1578-83.

18. Hill AD, Fowler RA, Burns KEA, Rose L, Pinto RL, Scales DC. Long-Term Outcomes and Health Care Utilization after Prolonged Mechanical Ventilation. Ann Am Thorac Soc. 2017;14:355-62.

19. Litwinowicz R, Bartus K, Drwila R, Kapelak B, Konstanty-Kalandyk J, Sobczynski R, et al. In-hospital mortality in cardiac surgery patients after readmission to the intensive care unit: a single-center experience with 10,992 patients. J Cardiothorac Vasc Anesth. 2015;29:570-5.

20. ARDS Definition Task Force, Ranieri VM, Rubenfeld GD, Thompson BT, Ferguson ND, Caldwell E, et al. Acute respiratory distress syndrome: the Berlin Definition. JAMA. 2012;307:2526-33.

21. Esteve F, Lopez-Delgado JC, Javierre C, Skaltsa K, Carrio ML, Rodríguez-Castro D, et al. Evaluation of the PaO2/FiO2 ratio after cardiac surgery as a predictor of outcome during hospital stay. BMC Anesthesiol. 2014;14:83.

22. Ranucci M, Ballotta A, La Rovere MT, Castelvecchio S, Surgical and Clinical Outcome Research (SCORE) Group. Postoperative hypoxia and length of intensive care unit stay after cardiac surgery: the underweight paradox? PLoS One. 2014;9:e93992.

23. Olper L, Bignami E, Di Prima AL, Albini S, Nascimbene S, Cabrini L, et al. Continuous Positive Airway Pressure Versus Oxygen Therapy in the Cardiac Surgical Ward: A Randomized Trial. J Cardiothorac Vasc Anesth. 2017;31:115-21.

24. Ladha K, Vidal Melo MF, McLean DJ, Wanderer JP, Grabitz SD, Kurth T, et al. Intraoperative protective mechanical ventilation and risk of postoperative respiratory complications: hospital based registry study. BMJ. 2015;351:h3646.

25. DeLong ER, DeLong DM, Clarke-Pearson DL. Comparing the areas under two or more correlated receiver operating characteristic curves: a nonparametric approach. Biometrics. 1988;44:837-45.

26. World Medical Association. Declaration of Helsinki: Ethical Principles for Medical Research Involving Human Subjects, October 2008. Canary Press; 2008.

27. Rhodes A, Evans LE, Alhazzani W, Levy MM, Antonelli M, Ferrer R, et al. Surviving Sepsis Campaign: International Guidelines for Management of Sepsis and Septic Shock: 2016. Intensive Care Med. 2017;43:304-77.

28. Ranucci M, Castelvecchio S, Conte M, Megliola G, Speziale G, Fiore F, et al. The easier, the better: Age, creatinine, ejection fraction score for operative mortality risk stratification in a series of 29,659 patients undergoing elective cardiac surgery. J Thorac Cardiovasc Surg. 2011;142:581-6.

29. Kapadohos T, Angelopoulos E, Vasileiadis I, Nanas S, Kotanidou A, Karabinis A, et al. Determinants of prolonged intensive care unit stay in patients after cardiac surgery: a prospective observational study. J Thorac Dis. 2017;9:70-9.

\section{Figures}




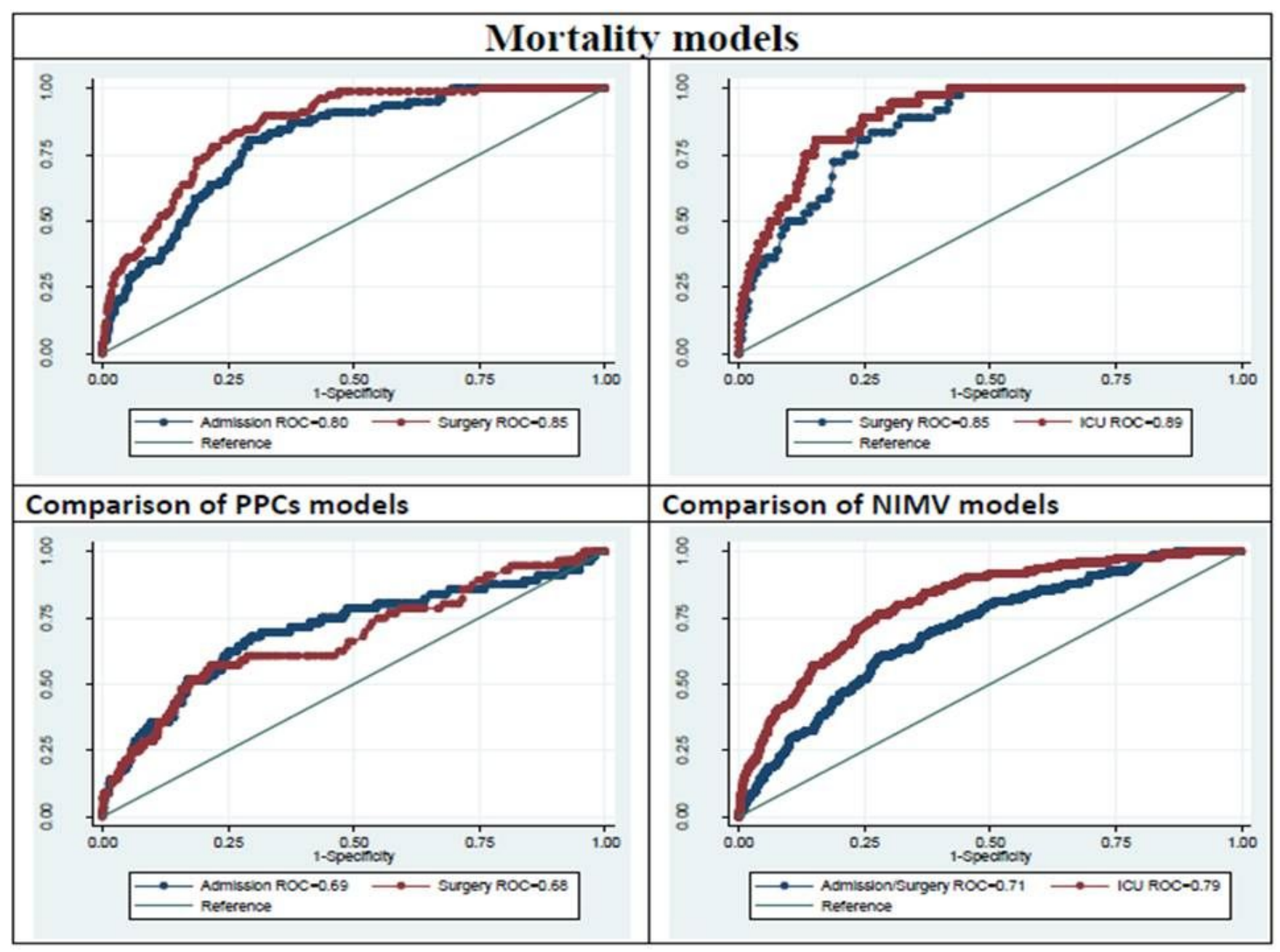

Figure 1

Comparison between predictive models by roc curves.

Page $11 / 12$ 


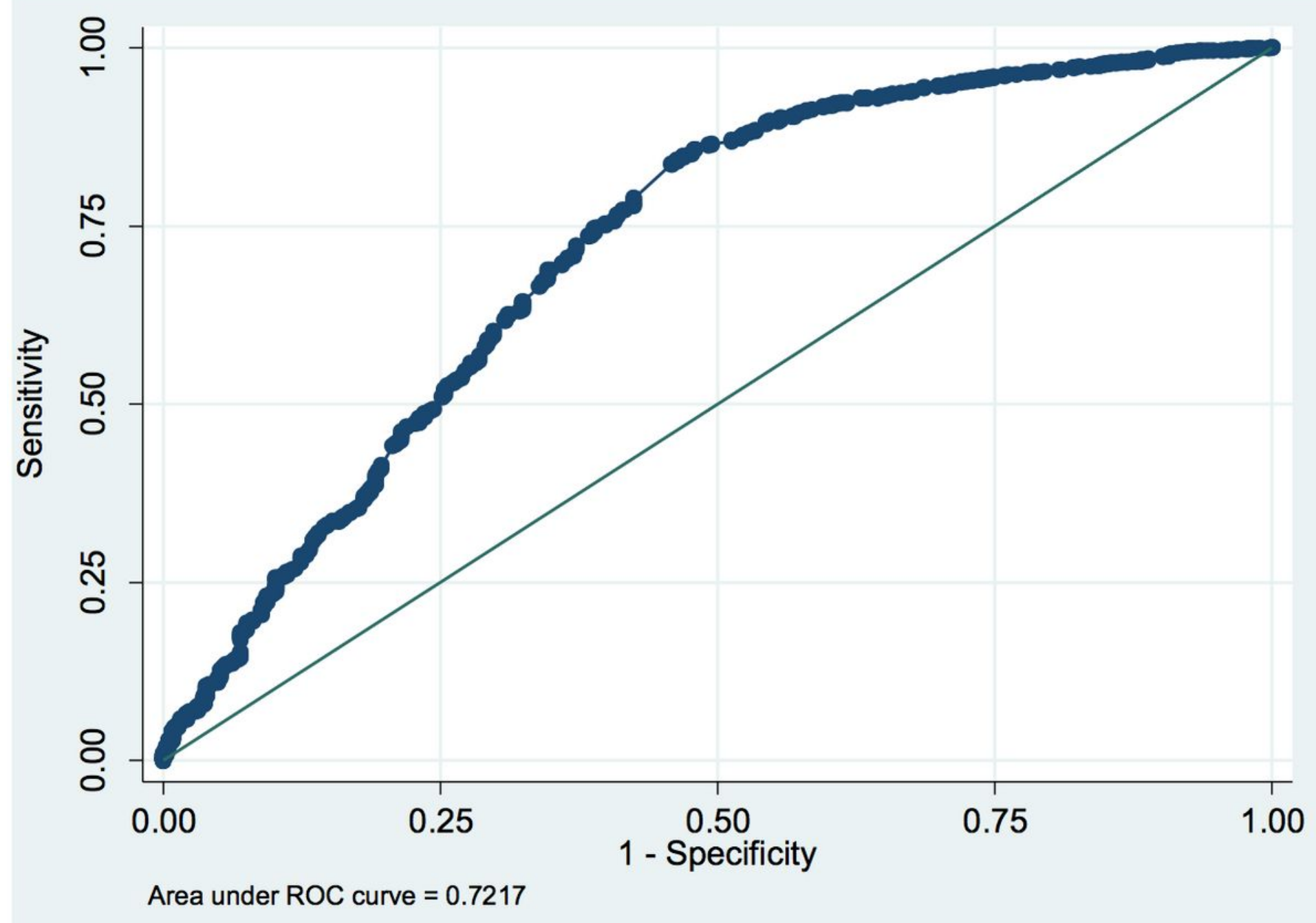

Figure 2

The roc curve for the $\mathrm{PaO} 2 / \mathrm{FiO} 2$ ratio and the incidence of post-operative non invasive mechanical ventilation.

\section{Supplementary Files}

This is a list of supplementary files associated with this preprint. Click to download.

- Supplementarymaterial.docx 